Pacific Journal of Mathematic 


\title{
ON A CLASS OF UNBOUNDED OPERATOR ALGEBRAS III
}

\author{
Atsushi INOUE
}

In this paper we continue our study of unbounded operator algebras begun in previous papers. In particular, the unbounded Hilbert algebras are studied. The primary purpose of this paper is to give necessary and sufficient conditions under which an unbounded Hilbert algebra is pure.

1. Introduction. In the previous paper [6] we began our study of unbounded Hilbert algebras and raised the following problem.

Problem. Let $\mathscr{D}_{0}$ be a maximal Hilbert algebra in a Hilbert space $\mathfrak{S}$. Does there exist a pure unbounded Hilbert algebra over $\mathscr{D}_{0}$ in $\mathfrak{S}$ ?

In this paper we find that if $\mathscr{D}_{0} \neq \mathscr{S}_{\mathcal{C}}$ then the answer is affirmative. That is, if $\mathscr{D}_{0} \neq \mathfrak{S}$, then the maximal unbounded Hilbert algebra $L_{2}^{\omega}\left(\mathscr{D}_{0}\right)$ is a pure unbounded Hilbert algebra over $\mathscr{D}_{0}$ in $\mathfrak{S}$. It therefore seems that our study of a class of unbounded operator algebras called $E W^{\sharp}$-algebras is significant. For, from ([6] Theorem $3.10)$ if $\mathscr{D}_{0} \neq \mathscr{S}$ then there necessarily exist pure $E W^{\sharp}$-algebras over the left von Neumann algebra $\mathscr{U}_{0}\left(\mathscr{D}_{0}\right)$ of $\mathscr{D}_{0}$ and if $\mathfrak{U}_{0}$ is a semifinite von Neumann algebra with a faithful normal semifinite trace $\varphi_{0}$ on $\mathfrak{A}_{0}^{+}$and $L^{2}\left(\varphi_{0}\right) \neq \mathfrak{U}_{0} \cap L^{2}\left(\varphi_{0}\right)$, then there exist pure $E W^{*}$-algebras over $\mathfrak{A}_{0}$ such that are isomorphic to standard $E W^{\sharp}$-algebras.

2. Basic theory for unbounded Hilbert algebras. We give here only the basic definitions and facts needed. For a more complete discussion of the basic properties of unbounded Hilbert algebras the reader is referred to $[6,7]$.

Let $\mathscr{D}$ be a pre-Hilbert space with an inner product [|] and be a *-algebra. Let $\mathfrak{S}$ be the completion of $\mathscr{D}$. Suppose that $\mathscr{D}$ satisfies;

$$
\begin{array}{cl}
(\xi \mid \eta) & =\left(\eta^{*} \mid \xi^{*}\right), \quad \xi, \eta \in \mathscr{D}, \\
(\xi \eta \mid \zeta) & =\left(\eta \mid \xi^{*} \zeta\right), \quad \xi, \eta, \zeta \in \mathscr{D} .
\end{array}
$$

Now, we define $\pi(\xi)$ and $\pi^{\prime}(\xi)$ by;

$$
\pi(\xi) \eta=\xi \eta \quad \text { and } \pi^{\prime}(\xi) \eta=\eta \xi, \quad \eta \in \mathscr{D} .
$$

Then, by (2), we know that $\pi(\xi)$ and $\pi^{\prime}(\xi)$ are closable operators on $\mathfrak{S}$ with the domain $\mathscr{D}$ and $\pi(\xi)^{*} \supset \pi\left(\xi^{*}\right), \pi^{\prime}(\xi)^{*} \supset \pi^{\prime}\left(\xi^{*}\right)$. 
Definition 2.1. If $\mathscr{D}$ satisfies (1), (2) and (3) $\mathscr{D}_{0}^{2}$ is dense in Sc, where

$$
\begin{array}{r}
\mathscr{D}_{0}=\{\xi \in \mathscr{D} ; \pi(\xi) \text { is continuous with respect to the } \\
\text { pre-Hilbert space structure of } \mathscr{D}\},
\end{array}
$$

then $\mathscr{D}$ is called an unbounded Hilbert algebra over $\mathscr{D}_{0}$ in $\mathscr{S}$ and $\pi\left(\right.$ resp. $\left.\pi^{\prime}\right)$ is called the left (resp. right) regular representation of $\mathscr{D}$. In particular, if $\mathscr{D}_{0} \neq \mathscr{D}$, then $\mathscr{D}$ is called pure.

Let $\mathscr{D}$ be an unbounded Hilbert algebra over $\mathscr{D}_{0}$ and let $\mathscr{S}$ be the completion of $\mathscr{D}$. Clearly $\mathscr{D}_{0}$ is a Hilbert algebra and the completion of $\mathscr{D}_{0}$ is the Hilbert space $\mathfrak{S}_{\text {. }}$ Let $\pi\left(\right.$ resp. $\left.\pi^{\prime}\right)$ be the left (resp. right) regular representation of $\mathscr{D}$ and let $\pi_{0}\left(\right.$ resp. $\left.\pi_{0}^{\prime}\right)$ be the left (resp. right) regular representation of the Hilbert algebra $\mathscr{D}_{0}$.

Let $\mathfrak{A}$ be a family of closable operators on a Hilbert space. Then we denote by $\bar{A}$ the closure of $A \in \mathfrak{A}$ and put $\overline{\mathfrak{A}}=\{\bar{A} ; A \in \mathfrak{X}\}$.

For each $x \in \mathfrak{F}$ we denote $\pi_{0}(x)$ and $\pi_{0}^{\prime}(x)$ by;

$$
\pi_{0}(x) \xi=\overline{\pi_{0}^{\prime}(\xi)} x, \quad \pi_{0}^{\prime}(x) \xi=\overline{\pi_{0}(\xi)} x, \quad \xi \in \mathscr{D}_{0} .
$$

Then $\pi_{0}(x)$ and $\pi_{0}^{\prime}(x)$ are linear operators on $\mathscr{S}$ with the domain $\mathscr{D}_{0}$. The involution on $\mathscr{D}$ is extended to an involution on $\mathfrak{S}$, which is also denoted by $*$. Then we have $\overline{\pi_{0}\left(x^{*}\right)}=\pi_{0}(x)^{*}$ and $\overline{\pi_{0}^{\prime}\left(x^{*}\right)}=\pi_{0}^{\prime}(x)^{*}$.

Lemma 2.2. (1) For each $\xi \in \mathscr{D}$ we have

$$
\begin{aligned}
& \overline{\pi(\xi)}=\overline{\pi_{0}(\xi)}, \quad \overline{\pi^{\prime}(\xi)}=\overline{\pi_{0}^{\prime}(\xi)}, \\
& \overline{\pi\left(\xi^{*}\right)}=\pi(\xi)^{*}, \quad \overline{\pi^{\prime}\left(\xi^{*}\right)}=\pi^{\prime}(\xi)^{*} .
\end{aligned}
$$

(2) For each $\lambda \in C$ (the field of complex numbers) and $\xi, \eta \in \mathscr{D}$ we have

$$
\begin{aligned}
& \overline{\pi(\xi)}+\overline{\pi(\eta)}:=\overline{\overline{\pi(\xi)}+\overline{\pi(\eta)}}=\overline{\pi(\xi+\eta)}, \\
& \overline{\pi(\xi)} \cdot \overline{\pi(\eta)}:=\overline{\overline{\pi(\xi)} \overline{\pi(\eta)}}=\overline{\pi(\xi \eta)}, \\
& \lambda \cdot \overline{\pi(\xi):}=\left\{\begin{array}{ccc}
\lambda \overline{\pi(\xi)}, & \text { if } \lambda \neq 0 \\
0, & \text { if } \lambda=0
\end{array}=\overline{\pi(\lambda \xi)}, \quad \pi(\xi)^{*}=\overline{\pi\left(\xi^{*}\right)} .\right.
\end{aligned}
$$

Therefore $\overline{\pi(\mathscr{D})}$ is a *-algebra of closed operators on $\mathfrak{F}$ under the operations of strong sum, strong product, adjoint and strong scalar multiplication. Similarly $\overline{\pi^{\prime}(\mathscr{D})}$ is a *-algebra of closed operators on $\mathfrak{S}$. 
Let $\mathscr{L}_{0}\left(\mathscr{D}_{0}\right)$ (resp. $\mathscr{\mathscr { V }}_{0}\left(\mathscr{D}_{0}\right)$ ) be the left (resp. right) von Neumann algebra of the Hilbert algebra $\mathscr{D}_{0}$ and let $\varphi_{0}$ be the natural trace on $\mathscr{U}_{0}\left(\mathscr{D}_{0}\right)^{+}$. Let $\mathfrak{B}(\mathfrak{S})$ be the set of all bounded linear operators on S. Putting

$$
\left(\mathscr{D}_{0}\right)_{b}=\left\{x \in \mathfrak{S} ; \overline{\pi_{0}(x)} \in \mathfrak{B}(\mathfrak{S})\right\},
$$

$\left(\mathscr{D}_{0}\right)_{b}$ is a Hilbert algebra containing $\mathscr{D}_{0}$. If $\mathscr{D}_{0}=\left(\mathscr{D}_{0}\right)_{b}$, then $\mathscr{D}_{0}$ is called a maximal Hilbert algebra in $\mathfrak{K}$.

Let $\mathfrak{M}$ be the set of all measurable operators on $\mathfrak{S}$ with respect to $\mathscr{U}_{0}\left(\mathscr{D}_{0}\right)$. For every $T \in \mathfrak{M}^{+}$we put

$$
\mu_{0}(T)=\sup \left[\varphi_{0}\left(\overline{\pi_{0}(\xi)}\right) ; 0 \leqq \overline{\pi_{0}(\xi)} \leqq T, \xi \in\left(\mathscr{D}_{0}\right)_{b}^{2}\right]
$$

and

$$
L^{p}\left(\varphi_{0}\right)=\left\{T \in \mathfrak{M} ;\|T\|_{p}:=\mu_{0}\left(|T|^{p}\right)^{1 / p}<\infty\right\}, \quad 1 \leqq p<\infty .
$$

Then $\|T\|_{p}$ is called the $L^{p}$-norm of $T$ in $L^{p}\left(\varphi_{0}\right)$ and $\mu_{0}$ is called the integral on $L^{1}\left(\varphi_{0}\right)$. If $p=\infty$, we shall identify $\mathscr{C}_{0}\left(\mathscr{D}_{0}\right)$ with $L^{\infty}\left(\varphi_{0}\right)$ and we denote by $\|T\|$ or $\|T\|_{\infty}$ the operator norm of $T \in \mathscr{U}_{0}\left(\mathscr{D}_{0}\right)$.

Definition 2.3. We define $L^{\omega}$-spaces with respect to $\varphi_{0}$ and $\mathscr{D}_{0}$ as follows;

$$
L^{\omega}\left(\varphi_{0}\right)=\bigcap_{1 \leqq p<\infty} L^{p}\left(\varphi_{0}\right), \quad L_{2}^{\omega}\left(\varphi_{0}\right)=\bigcap_{2 \leqq p<\infty} L^{p}\left(\varphi_{0}\right),
$$

and

$$
L^{\omega}\left(\mathscr{D}_{0}\right)=\left\{x \in \mathfrak{S} ; \overline{\pi_{0}(x)} \in L^{\omega}\left(\varphi_{0}\right)\right\}, \quad L_{2}^{\omega}\left(\mathscr{D}_{0}\right)=\left\{x \in \mathfrak{S} ; \overline{\pi_{0}(x)} \in L_{2}^{\omega}\left(\varphi_{0}\right)\right\},
$$

respectively. For $p \geqq 2$ we set

$$
\begin{gathered}
L_{2}^{p}\left(\mathscr{D}_{0}\right)=\left\{x \in \mathfrak{S}_{\mathcal{C}} ; \overline{\pi_{0}(x)} \in L^{p}\left(\varphi_{0}\right)\right\}, \\
\|x\|_{p}=\left\|\overline{\pi_{0}(x)}\right\|_{p}, \quad x \in L_{2}^{p}\left(\mathscr{D}_{0}\right) \\
\|x\|_{\infty}=\left\|\overline{\pi_{0}(x)}\right\|_{\infty}, \quad x \in L_{2}^{\infty}\left(\mathscr{D}_{0}\right)=\left(\mathscr{D}_{0}\right)_{b} .
\end{gathered}
$$

TheOREM 2.4. $L_{2}^{\omega}\left(\mathscr{D}_{0}\right)$ (resp. $L^{\omega}\left(\mathscr{D}_{0}\right)$ ) is an unbounded Hilbert algebra over $\left(\mathscr{D}_{0}\right)_{b}$ (resp. $\left.\left(\mathscr{D}_{0}\right)_{b}^{2}\right)$ in $\mathfrak{K}_{\mathcal{E}}$. If $\mathscr{D}$ is a pure unbounded Hilbert algebra, then $\mathscr{D}$ is a *-subalgebra of $L_{2}^{\omega}\left(\mathscr{D}_{0}\right)$. Hence $L_{2}^{\omega}\left(\mathscr{D}_{0}\right)$ is maximal among unbounded Hilbert algebras containing $\mathscr{D}_{0}$

Proof. ([6] Theorem 3.9)

3. Necessary and sufficient conditions under which $L_{2}^{\omega}\left(\mathscr{D}_{0}\right)$ is pure. Let $\mathscr{D}_{0}$ be a Hilbert algebra in a Hilbert space $\mathscr{S}$ and let $\varphi_{0}$ be the natural trace on $\mathscr{U}_{0}\left(\mathscr{D}_{0}\right)^{+}$. 
LEMMA 3.1. For $2 \leqq p<q$ we have

$$
L_{2}^{2}\left(\mathscr{D}_{0}\right)=\mathscr{K} \supset L_{2}^{p}\left(\mathscr{D}_{0}\right) \supset L_{2}^{q}\left(\mathscr{D}_{0}\right) \supset L_{2}^{\omega}\left(\mathscr{D}_{0}\right) \supset L_{2}^{\infty}\left(\mathscr{D}_{0}\right)=\left(\mathscr{D}_{0}\right)_{b}
$$

and

$$
L_{2}^{\omega}\left(\mathscr{D}_{0}\right)=\bigcap_{2 \leqq n<\infty} L_{2}^{n}\left(\mathscr{D}_{0}\right),
$$

where $n$ is an integer.

Proof. For each $x \in L_{2}^{q}\left(\mathscr{D}_{0}\right)$ let $\overline{\pi_{0}(x)}=U\left|\overline{\pi_{0}(x)}\right|$ be the polar decomposition and let $\left|\overline{\pi_{0}(x)}\right|=\int_{0}^{\infty} \lambda d E(\lambda)$ be the spectral resolution of $\left|\overline{\pi_{0}(x)}\right|$. Then,

$$
\begin{aligned}
\|x\|_{p}^{p} & =\left\|\overline{\pi_{0}(x)}\right\|_{p}^{p}=-\int_{0}^{\infty} \lambda^{p} d \varphi_{0}\left(E(\lambda)^{\perp}\right) \\
& =-\int_{0}^{1} \lambda^{p} d \varphi_{0}\left(E(\lambda)^{\perp}\right)-\int_{1}^{\infty} \lambda^{p} d \varphi_{0}\left(E(\lambda)^{\perp}\right) \\
& \leqq-\int_{0}^{1} \lambda^{2} d \varphi_{0}\left(E(\lambda)^{\perp}\right)-\int_{1}^{\infty} \lambda^{q} d \varphi_{0}\left(E(\lambda)^{\perp}\right) \\
& \leqq\|x\|_{2}^{2}+\|x\|_{q}^{q}<\infty .
\end{aligned}
$$

Hence, $x \in L_{2}^{p}\left(\mathscr{D}_{0}\right)$. Consequently $L_{2}^{p}\left(\mathscr{D}_{0}\right) \supset L_{2}^{q}\left(\mathscr{D}_{0}\right)$, and so we can easily show that $L_{2}^{\omega}\left(\mathscr{D}_{0}\right)=\bigcap_{2 \leqq n<\infty} L_{2}^{n}\left(\mathscr{D}_{0}\right)$ ( $n$; integer).

LEMmA 3.2. If $L_{2}^{p}\left(\mathscr{D}_{0}\right)=L_{2}^{q}\left(\mathscr{D}_{0}\right)$ for some $q>p \geqq 2$, then $L_{2}^{r}\left(\mathscr{D}_{0}\right)=L_{2}^{\omega}\left(\mathscr{D}_{0}\right)$ for all $r \in[p, \infty)$.

Proof. Let $x \in L_{2}^{p}\left(\mathscr{D}_{0}\right)=L_{2}^{q}\left(\mathscr{D}_{0}\right)$. Then, $\left|\overline{\pi_{0}(x)}\right|^{q / p} \in L^{p}\left(\varphi_{0}\right)$. Since $2<2 q / p \leqq q$ and $L_{2}^{2}\left(\mathscr{D}_{0}\right) \supset L_{2}^{2 q / p}\left(\mathscr{D}_{0}\right) \supset L_{2}^{q}\left(\mathscr{D}_{0}\right)$ (by Lemma 3.1), we get $x \in L_{2}^{2 q / p}\left(\mathscr{D}_{0}\right)$, i.e., $\left|\overline{\pi_{0}(x)}\right|^{2 q / p} \in L^{1}\left(\varphi_{0}\right)$. Hence, $\left|\overline{\pi_{0}(x)}\right|^{q / p} \in L^{p}\left(\varphi_{0}\right) \cap L^{2}\left(\varphi_{0}\right)$. Repeating the same argument, we get that $\left|\overline{\pi_{0}(x)}\right|^{(q / p){ }^{n}} \in L^{p}\left(\varphi_{0}\right) \cap$ $L^{2}\left(\mathscr{D}_{0}\right)(n=1,2, \cdots)$. From $q / p>1$ and Lemma 3.1, $x \in L_{2}^{\omega}\left(\mathscr{D}_{0}\right)$.

DEFINITION 3.3. An element $e$ of $\mathscr{D}_{0}$ is called a projection if $e^{2}=e=e^{*}$. Let $E\left(\mathscr{D}_{0}\right)$ denote the collection of all projections in $\mathscr{D}_{0}$.

THEOREM 3.4. Let $\mathscr{D}_{0}$ be a Hilbert algebra in $\mathfrak{S}_{\text {. }}$ Then the following conditions are equivalent.

(1) $L_{2}^{\omega}\left(\mathscr{D}_{0}\right)$ is pure.

(2) $L^{\omega}\left(\mathscr{D}_{0}\right)$ is pure.

(3) There exists a sequence $\left\{e_{n}\right\}$ of nonzero mutually orthogonal projections in $\left(\mathscr{D}_{0}\right)_{b}$ such that $\sum_{n=1}^{\infty}\left\|e_{n}\right\|_{2}^{2}<\infty$.

(4) $\mathfrak{S}$ is not a Hilbert algebra, i.e., $\left(\mathscr{D}_{0}\right)_{b} \neq \mathfrak{S}$.

(5) $L_{2}^{\omega}\left(\mathscr{D}_{0}\right) \neq \mathfrak{S}$. 
(6) $L_{2}^{p}\left(\mathscr{D}_{0}\right) \neq L_{2}^{q}\left(\mathscr{D}_{0}\right)$ for some $2 \leqq p<q$.

( 7 ) $L_{2}^{p}\left(\mathscr{D}_{0}\right) \neq L_{2}^{2}\left(\mathscr{D}_{0}\right)$ for each $p>2$.

In particular, if $\mathscr{D}_{0}$ has an identity, then (1) (7) are eqvivalent to $(7)^{\prime}$;

$(7)^{\prime} \quad L^{p}\left(\varphi_{0}\right) \neq L^{q}\left(\varphi_{0}\right)$ for each $q>p \geqq 1$.

Proof. From Lemma 3.1, for $2 \leqq p<q$

$$
L_{2}^{2} \supset L_{2}^{p} \supset L_{2}^{q} \supset L_{2}^{\omega} \supset\left(\mathscr{D}_{0}\right)_{b} \text { • }
$$

Hence, $(7) \Rightarrow(6) \Longrightarrow(5) \Longrightarrow(4)$ and $(2) \Longrightarrow(1)$ are easily showed.

(1) $\Rightarrow(7)$; If $L_{2}^{p}\left(\mathscr{D}_{0}\right)=L_{2}^{2}\left(\mathscr{D}_{0}\right)$ for some $p>2$, then from Lemma 3.2 we have $L_{2}^{2}\left(\mathscr{D}_{0}\right)=L_{2}^{\omega}\left(\mathscr{D}_{0}\right)$. Since $L_{2}^{\omega}\left(\mathscr{D}_{0}\right)$ is an algebra, for each $x \in L_{2}^{\omega}\left(\mathscr{D}_{0}\right), \mathscr{D}\left(\overline{\pi_{0}(x)}\right)=\mathfrak{S}_{\mathcal{E}}$, i.e., $\overline{\pi_{0}(x)} \in \mathfrak{B}\left(\mathfrak{S}_{\mathcal{C}}\right)$. Hence $L_{2}^{\omega}\left(\mathscr{D}_{0}\right)$ is a Hilbert algebra.

$(4) \Rightarrow(3)$; Suppose that $x \in \mathfrak{S}-\left(\mathscr{D}_{0}\right)_{b}$. Let $\left|\overline{\pi_{0}(x)}\right|=\int_{0}^{\infty} \lambda d E(\lambda)$ be the spectral resolution of $\left|\overline{\pi_{0}(x)}\right|$. Since $\left|\overline{\pi_{0}(x)}\right| \notin \mathfrak{B}(\mathfrak{S}), E(n+1)-$ $E(n) \neq 0$ for infinite many $n$, and so we may suppose that $E(n+1)-$ $E(n) \neq 0 \quad(n=1,2, \cdots)$. We shall show that $E(n+1)-E(n) \epsilon$ $L^{\infty}\left(\varphi_{0}\right) \cap L^{2}\left(\varphi_{0}\right)$. Clearly, $E(n+1)-E(n) \in L^{\infty}\left(\varphi_{0}\right)=\mathscr{U}_{0}\left(\mathscr{D}_{0}\right)$. Moreover, we have

$$
\begin{aligned}
\|E(n+1)-E(n)\|_{2}^{2} & =\varphi_{0}(E(n+1)-E(n))=-\int_{n}^{n+1} d \varphi_{0}\left(E(\lambda)^{\perp}\right) \\
& \leqq-\int_{n}^{n+1} \lambda^{2} d \varphi_{0}\left(E(\lambda)^{\perp}\right) \leqq\left\|\frac{\pi_{0}(x)}{\|_{2}^{2}}=\right\| x \|_{2}^{2} .
\end{aligned}
$$

Hence, $E(n+1)-E(n) \in L^{2}\left(\varphi_{0}\right) \quad(n=1,2, \cdots)$, and so there exists $e_{n} \in\left(\mathscr{D}_{0}\right)_{b}$ such that $E(n+1)-E(n)=\overline{\pi_{0}\left(e_{n}\right)}(n=1,2 \cdots)$. Clearly $\left\{e_{n}\right\}$ is a sequence of nonzero mutually orthogonal projections in $\left(\mathscr{D}_{0}\right)_{b}$. We shall show that $\sum_{n=1}^{\infty}\left\|e_{n}\right\|_{2}^{2}<\infty$. In fact, for $m>n$

$$
\begin{aligned}
\sum_{k=n}^{m}\left\|e_{k}\right\|_{2}^{2} & =\sum_{k=n}^{m} \varphi_{0}\left(\overline{\pi_{0}\left(e_{k}\right)}\right)=\sum_{k=n}^{m} \varphi_{0}(E(k+1)-E(k)) \\
& =\varphi_{0}(E(m+1)-E(n))
\end{aligned}
$$

and $\{E(m+1)-E(n)\}$ converges $\sigma$-weakly to $0(n, m \rightarrow \infty)$. Since $\varphi_{0}$ is $\sigma$-weakly continuous, we have

$$
\lim _{m, n \rightarrow \infty} \sum_{k=n}^{m}\left\|e_{k}\right\|_{2}^{2}=\lim _{m, n \rightarrow \infty} \varphi_{0}(E(m+1)-E(n))=0 .
$$

Hence, $\sum_{n=1}^{\infty}\left\|e_{n}\right\|_{2}^{2}<\infty$.

$(3) \Rightarrow(2)$; For some positive integer $k_{0}, \sum_{n=k_{0}}^{\infty}\left\|e_{n}\right\|_{2}^{2}<1$. We set 


$$
\begin{aligned}
& a_{0}=\sum_{n=k_{0}}^{\infty}\left\|e_{n}\right\|_{2}^{2}, \quad a_{1}=\sum_{n=k_{0}+1}^{\infty}\left\|e_{n}\right\|_{2}^{2}, \cdots, \quad a_{n}=\sum_{k=k_{0}+n}^{\infty}\left\|e_{k}\right\|_{2}^{2}, \cdots, \\
& b_{0}=\left|\log a_{0}\right|, \cdots, \quad b_{n}=\left|\log a_{n}\right|, \cdots
\end{aligned}
$$

and

$$
x=\sum_{n=0}^{\infty} b_{n} e_{k_{0}+n}
$$

We shall show that $x \in L^{\omega}\left(\mathscr{D}_{0}\right)-\left(\mathscr{D}_{0}\right)_{b}$. For every $p \in[1, \infty)$

$$
\sum_{n=0}^{\infty}\left|b_{n}\right|^{p}\left\|e_{k_{0}+n}\right\|_{2}^{2}<\int_{0}^{1}|\log x|^{p} d x=p !
$$

and so

$$
\lim _{m, n \rightarrow \infty}\left\|\sum_{k=n}^{m} b_{k} e_{k_{0}+k}\right\|_{2}^{2}=\lim _{m, n \rightarrow \infty} \sum_{k=n}^{m}\left|b_{k}\right|^{2}\left\|e_{k_{0}+k}\right\|_{2}^{2}=0 .
$$

Hence, $\quad x \in \mathscr{S}$ and $\|x\|_{2}^{2}=\sum_{n=0}^{\infty}\left|b_{n}\right|^{2}\left\|e_{k_{0}+n}\right\|_{2}^{2}$. Similarly, for every $p \in[1, \infty), \quad x \in L_{2}^{p}\left(\mathscr{D}_{0}\right)$ and $\|x\|_{p}^{p}=\sum_{n=0}^{\infty}\left|b_{n}\right|^{p}\left\|e_{k_{0}+n}\right\|_{2}^{2}$. Therefore, $x \in L^{\omega}\left(\mathscr{D}_{0}\right)$. On the other hand, $\lim _{n \rightarrow \infty} b_{n}=\infty$ and $\left\|e_{k_{0}+n}\right\|_{2}^{2} \neq 0$ $(n=1,2, \cdots)$, and so $\overline{\pi_{0}(x)} \notin \mathfrak{B}(\mathfrak{S})$. Hence, $x \in L^{\omega}\left(\mathscr{D}_{0}\right)-\left(\mathscr{D}_{0}\right)_{b}$. That is, $L^{\omega}\left(\mathscr{D}_{0}\right)$ is pure.

Suppose that $\mathscr{D}_{0}$ has an identity.

$(7)^{\prime} \Longrightarrow(7) ;$ Obvious.

$(7) \Longrightarrow(7)^{\prime} ;$ For $1 \leqq p<q$ we have

$$
L^{1}\left(\varphi_{0}\right) \supset L^{p}\left(\varphi_{0}\right) \supset L^{q}\left(\varphi_{0}\right) \supset L^{\omega}\left(\varphi_{0}\right) \supset L^{\infty}\left(\varphi_{0}\right) \text {. }
$$

Suppose that $L^{p}\left(\varphi_{0}\right)=L^{q}\left(\varphi_{0}\right)$ for $1 \leqq p<q$. Let $T \in L^{1}\left(\varphi_{0}\right)$. Then, $|T|^{1 / p} \in L^{p}\left(\varphi_{0}\right)=L^{q}\left(\varphi_{0}\right)$. Hence, $|T|^{q / p} \in L^{1}\left(\varphi_{0}\right)$. Repeating the same argument, $|T|^{(q / p)^{n}} \in L^{1}\left(\varphi_{0}\right)(n=1,2, \cdots)$, and so $|T| \in L^{(q / p)^{n}}\left(\varphi_{0}\right)$ $(n=1,2, \cdots)$. From $q / p>1$ and Lemma 3.1, $|T| \in L^{\omega}\left(\varphi_{0}\right)$, and so $T \in L^{\omega}\left(\varphi_{0}\right)$.

Let $\mathscr{D}_{0}$ be a Hilbert algebra in $\mathfrak{S}_{\text {. }}$ From Theorem 3.4 , if $\mathfrak{S}$ is not a Hilbert algebra, i.e., $\left(\mathscr{D}_{0}\right)_{b} \neq \mathscr{S}_{\mathcal{c}}$, then $L_{2}^{\omega}\left(\mathscr{D}_{0}\right)$ becomes a pure unbounded Hilbert algebra over $\left(\mathscr{D}_{0}\right)_{b}$ in $\mathfrak{S}_{\mathcal{C}}$. So, the previous problem is solved. If $L_{2}^{\omega}\left(\mathscr{D}_{0}\right)$ is a Hilbert algebra, then $\mathfrak{S}_{\mathcal{C}}$ is a Hilbert algebra and $L_{2}^{\omega}\left(\mathscr{D}_{0}\right)=\mathfrak{S}_{\mathrm{C}}$. Hence we can give some conditions for $L_{2}^{\omega}\left(\mathscr{D}_{0}\right)$ to be a Hilbert algebra.

CoROLLARY 3.5. Let $\mathscr{D}_{0}$ be a Hilbert algebra in $\mathfrak{S}$. Then the following conditions are equivalent.

(1) $\mathfrak{S}$ is a Hilbert algebra.

(2) $L_{2}^{\omega}\left(\mathscr{D}_{0}\right)$ is a Hilbert algebra. 
(3) $\mathfrak{S}=L_{2}^{\omega}\left(\mathscr{D}_{0}\right)=\left(\mathscr{D}_{0}\right)_{b}$.

(4) Either $E\left(\left(\mathscr{D}_{0}\right)_{b}\right)$ is a finite set or $\sum_{n=1}^{\infty}\left\|e_{n}\right\|_{2}^{2}=\infty$ for each sequence $\left\{e_{n}\right\}$ of mutually orthogonal projections in $E\left(\left(\mathscr{D}_{0}\right)_{b}\right)$.

(5) There exists $C>0$ such that $\|e\|_{2} \geqq C$ for all $e \in E\left(\left(\mathscr{D}_{0}\right)_{b}\right)$.

(6) $L_{2}^{p}\left(\mathscr{D}_{0}\right)=L_{2}^{q}\left(\mathscr{D}_{0}\right)$ for each $q>p \geqq 2$.

( 7 ) $L_{2}^{p}\left(\mathscr{D}_{0}\right)=L_{2}^{2}\left(\mathscr{D}_{0}\right)$ for some $p>2$.

In particular, if $\mathscr{D}_{0}$ has an identity, then $(1) \sim(7)$ are equivalent to $(7)^{\prime}$;

$$
(7)^{\prime} \quad L_{2}^{p}\left(\mathscr{D}_{0}\right)=L_{2}^{q}\left(\mathscr{D}_{0}\right) \text { for some } q>p \geqq 1 \text {. }
$$

Proof. From Theorem $3.4(1) \Leftrightarrow(2) \Leftrightarrow(3) \Leftrightarrow(6) \Leftrightarrow(7) \Leftrightarrow(7)^{\prime}$ are easily showed.

Let $E=\overline{\pi_{0}(e)}$ and $F=\overline{\pi_{0}(f)}$ for $e, f \in E\left(\left(\mathscr{D}_{0}\right)_{b}\right)$. We denote by $E \cap F$ (resp. $E \cup F$ ) the projection onto $E \mathfrak{S} \cap F \mathfrak{S}$ (resp. $E \mathfrak{S} \cup F \mathfrak{S}$ ). Clearly, $E \cap F$ and $E \cup F$ in $L^{\infty}\left(\varphi_{0}\right) \cap L^{2}\left(\varphi_{0}\right)$. Hence there exist projections $e \cap f$ and $e \cup f$ in $\left(\mathscr{D}_{0}\right)_{b}$ such that $E \cap F=\overline{\pi_{0}(e \cap f)}$ and $E \cup F=\overline{\pi_{0}(e \cup f)}$.

If $E\left(\left(\mathscr{D}_{0}\right)_{b}\right)$ is an infinite set, then there exists a sequence $\left\{e_{n}\right\}$ of mutually orthogonal projections in $E\left(\left(\mathscr{D}_{0}\right)_{b}\right)$. In fact, the following two cases are considered.

(i ) There exists a sequence $\left\{e_{n}\right\}$ of $E\left(\left(\mathscr{D}_{0}\right)_{b}\right)$ such that

$$
e_{2}-\left(e_{1} \cap e_{2}\right) \neq 0, \cdots, e_{n}-\left(e_{1} \cup e_{2} \cup \cdots \cup e_{n-1}\right) \cap e_{n} \neq 0, \cdots \text {. }
$$

(ii) There exists a sequence $\left\{e_{n}\right\}$ of $E\left(\left(\mathscr{D}_{0}\right)_{b}\right)$ such that $e_{1}>e_{n}$ for all $n \geqq 2$.

(i); Obvious.

(ii); We set

$$
\begin{aligned}
& p_{1}=e_{1}, \cdots, \quad p_{n}=e_{1}-\bigcup_{k=2}^{n} e_{k}, \cdots, \\
& q_{n}=p_{n}-p_{n+1}, \quad n=1,2, \cdots .
\end{aligned}
$$

If $q_{n} \neq 0$ for infinite many $n$, then $\left\{q_{n}\right\}$ is a sequence of mutually orthogonal projections in $E\left(\left(\mathscr{D}_{0}\right)_{b}\right)$. If $q_{n}=0$ for infinite many $n$, then $e_{n}>e_{n+1}$ for infinite many $n$. Putting $f_{n}=e_{n}-e_{n+1},\left\{f_{n}\right\}$ is a sequence of mutually orthogonal projections in $E\left(\left(\mathscr{D}_{0}\right)_{b}\right)$. From the above argument and Theorem $3.4,(2) \Leftrightarrow(4)$ is easily showed.

$(5) \Rightarrow(4)$; Obvious.

$(4) \Rightarrow(5)$; Suppose that (5) is not satisfied. For each $n$ there exists $e_{n} \in E\left(\left(\mathscr{D}_{0}\right)_{b}\right)$ such that $\left\|e_{n}\right\|_{2}<1 / n$. After a slight modification of the above, we can make a sequence $\left\{p_{n}\right\}$ of mutually orthogonal projections in $E\left(\left(\mathscr{D}_{0}\right)_{b}\right)$ such that $\sum_{n=1}^{\infty}\left\|p_{n}\right\|_{2}^{2} \leqq \sum_{n=1}^{\infty}\left\|e_{n}\right\|_{2}^{2} \leqq$ $\sum_{n=1}^{\infty} 1 / n^{2}<\infty$ 
4. Standard $E W^{\sharp}$-algebras. From ([6] Theorem 3.10) if $\mathscr{D}$ is a pure unbounded Hilbert algebra over $\mathscr{D}_{0}$, then there exists the pure $E W^{\sharp}$-algebra $\mathscr{U}(\mathscr{D})$ on $L_{2}^{\omega}\left(\mathscr{D}_{0}\right)$ over $\mathscr{U}_{0}\left(\mathscr{D}_{0}\right)$. So, from Theorem 3.4, if $\mathscr{D}_{0}$ is a Hilbert algebra in $\mathfrak{K}$ and $\left(\mathscr{D}_{0}\right)_{b} \neq \mathscr{S}_{\mathcal{E}}$, then there necessarily exist pure $E W^{\sharp}$-algebras over $\mathscr{U}_{0}\left(\mathscr{D}_{0}\right)$. Hence it seems that our study of $E W^{\sharp}$-algebras is significant. For a more complete discussion of the above argument we give here the basic definitions and facts of $E W^{\sharp}$-algebras.

Definition 4.1. Let $\mathfrak{D}$ be a pre-Hilbert space with an inner product $(\mid)$ and let $\mathscr{S}$ be the completion of $\mathscr{D}$. We denote the set of all linear operators on $\mathfrak{D}$ by $\mathfrak{L}(\mathfrak{D})$. A subalgebra $\mathfrak{A}$ of $\mathfrak{L}(\mathfrak{D})$ is called a $\#$-algebra on $\mathfrak{D}$ if there exists an involution on 2 ; $A \rightarrow A^{\sharp}$ such that

$$
(A \xi \mid \eta)=\left(\xi \mid A^{\sharp} \eta\right), \quad A \in \mathfrak{A}, \quad \xi, \eta \in \mathfrak{D} .
$$

We set

$$
\mathfrak{A}_{b}=\{A \in \mathfrak{X} ; \bar{A} \in \mathfrak{B}(\mathfrak{S})\} \text {. }
$$

Let $\mathfrak{A}$ be a $\#$-algebra on $\mathfrak{D}$ with an identity operator $I$. $\cdot \mathfrak{A}$ is called a symmetric \#-algebra on $\mathfrak{D}$ if $\left(I+A^{\sharp} A\right)^{-1}$ exists and lies in $\mathfrak{A}_{b}$ for every $A \in \mathfrak{A}$.

A symmetric \#-algebra $\mathfrak{A}$ on $\mathfrak{D}$ is said to be an $E W^{\sharp}$-algebra over $\overline{\mathfrak{A}}_{b}$ if $\overline{\mathfrak{A}}_{b}$ is a von Neumann algebra. If $\mathfrak{A} \neq \mathfrak{A}$, then $\mathfrak{A}$ is called a pure $E W^{\sharp}$-algebra.

Let $\mathfrak{A}$ be a set of densely-defined closed operators on $\mathfrak{F}$ which is $a *$-algebra under the operations of strong sum, strong product, adjoint and strong scalar multiplication. $\mathfrak{A}$ is said to be an $E W^{*}$ algebra over $\mathfrak{A}_{b}$ if $\left(I+T^{*} T\right)^{-1} \in \mathfrak{A}$ for every $T \in \mathfrak{A}$ and the sub-algebra $\mathfrak{A}_{b}$ of bounded operators in $\mathfrak{A}$ is a von Neumann algebra. If $\mathfrak{A} \neq \mathfrak{A}_{b}$, then $\mathfrak{A}$ is called a pure $E W^{*}$-algebra.

Clearly if $\mathfrak{A}$ is an (resp. pure) $E W^{\sharp}$-algebra, then $\overline{\mathfrak{A}}$ is an (resp. pure) $E W^{*}$-algebra.

Let $\mathscr{D}$ be an unbounded Hilbert algebra over $\mathscr{D}_{0}$ in a Hibert space $\mathscr{S}_{\mathcal{C}}$ and let $\varphi_{0}\left(\right.$ resp. $\left.\psi_{0}\right)$ be the natural trace on $\mathscr{U}_{0}\left(\mathscr{D}_{0}\right)^{+}$(resp. $\left.\mathscr{\mathscr { V }}_{0}\left(\mathscr{D}_{0}\right)^{+}\right)$. For every $x \in \mathscr{S}$ we see that

$$
\overline{J \pi_{0}(x)} J=\overline{\pi_{0}^{\prime}\left(x^{*}\right)} \text { and } \overline{\pi_{0}^{\prime}(x)} J=\overline{\pi_{0}\left(x^{*}\right)},
$$

where $J$ denotes the involution $*$ on $\mathfrak{S}$. Hence we get that

$$
\begin{aligned}
& J L^{\omega}\left(\varphi_{0}\right) J=L^{\omega}\left(\psi_{0}\right), \quad J L_{2}^{\omega}\left(\varphi_{0}\right) J=L_{2}^{\omega}\left(\psi_{0}\right), \\
& L^{\omega}\left(\mathscr{D}_{0}\right)=\left\{x \in \mathcal{S} ; \overline{\pi_{0}^{\prime}(x)} \in L^{\omega}\left(\psi_{0}\right)\right\}, \quad L_{2}^{\omega}\left(\mathscr{D}_{0}\right)=\left\{x \in \mathcal{F}_{\mathcal{C}} ; \overline{\pi_{0}^{\prime}(x)} \in L_{2}^{\omega}\left(\psi_{0}\right)\right\}
\end{aligned}
$$


and

$$
\mathscr{U}_{0}\left(\mathscr{D}_{0}\right) L_{2}^{\omega}\left(\mathscr{D}_{0}\right) \subset L_{2}^{\omega}\left(\mathscr{D}_{0}\right), \quad \mathscr{V}_{0}\left(\mathscr{D}_{0}\right) L_{2}^{\omega}\left(\mathscr{D}_{0}\right) \subset L_{2}^{\omega}\left(\mathscr{D}_{0}\right)
$$

Let $\pi_{2}^{(\omega}$ (resp. $\left.\left(\pi^{\prime}\right)_{2}^{\omega}\right)$ be the left (resp. right) regular representation of $L_{2}^{\omega}\left(\mathscr{D}_{0}\right)$ and let

$$
\begin{aligned}
& \mathscr{U}_{0}\left(\mathscr{D}_{0}\right) / L_{2}^{\omega}\left(\mathscr{D}_{0}\right)=\left\{T / L_{2}^{\omega}\left(\mathscr{D}_{0}\right) ; T \in \mathscr{U}_{0}\left(\mathscr{D}_{0}\right)\right\}, \\
& \mathscr{\mathscr { V }}_{0}\left(\mathscr{D}_{0}\right) / L_{2}^{\omega}\left(\mathscr{D}_{0}\right)=\left\{T^{\prime} / L_{2}^{\omega}\left(\mathscr{D}_{0}\right) ; T^{\prime} \in \mathscr{\mathscr { V }}_{0}\left(\mathscr{D}_{0}\right)\right\} \text {, }
\end{aligned}
$$

where $T / L_{2}^{\omega}\left(\mathscr{D}_{0}\right)$ is the restriction of $T$ onto $L_{2}^{\omega}\left(\mathscr{D}_{0}\right)$. Then $\pi_{2}^{\omega}(\mathscr{D})$, $\left(\pi^{\prime}\right)_{2}^{\omega}(\mathscr{D}), \mathscr{U}_{0}\left(\mathscr{D}_{0}\right) / L_{2}^{\omega}\left(\mathscr{D}_{0}\right)$ and $\mathscr{\mathscr { V }}_{0}\left(\mathscr{D}_{0}\right) / L_{2}^{\omega}\left(\mathscr{D}_{0}\right)$ are \#-algebras on $L_{2}^{\omega}\left(\mathscr{D}_{0}\right)$ under $\pi_{2}^{\omega}(\xi)^{\sharp}=\pi_{2}^{\omega}\left(\xi^{*}\right),\left(\pi^{\prime}\right)_{2}^{\omega}(\xi)^{\sharp}=\left(\pi^{\prime}\right)_{2}^{\omega}\left(\xi^{*}\right),\left(T / L_{2}^{\omega}\left(\mathscr{D}_{0}\right)\right)^{\sharp}=T^{*} / L_{2}^{\omega}\left(\mathscr{D}_{0}\right)$ and $\left(T^{\prime} / L_{2}^{\omega}\left(\mathscr{D}_{0}\right)\right)^{\sharp}=\left(T^{\prime}\right)^{*} / L_{2}^{\omega}\left(\mathscr{D}_{0}\right)$, respectively.

Notation. We denote by $\mathscr{U}(\mathscr{D})($ resp. $\mathscr{Y}(\mathscr{D}))$ the $\#$-algebra on $L_{2}^{\omega}\left(\mathscr{D}_{0}\right)$ generated by $\pi_{2}^{\omega}(\mathscr{D})\left(\right.$ resp. $\left.\left(\pi^{\prime}\right)_{2}^{\omega}(\mathscr{D})\right)$ and $\mathscr{U}_{0}\left(\mathscr{D}_{0}\right) / L_{2}^{\omega}\left(\mathscr{D}_{0}\right)$ (resp. $\left.\mathscr{V}_{0}\left(\mathscr{D}_{0}\right) / L_{2}^{\omega}\left(\mathscr{D}_{0}\right)\right)$.

THEOREM 4.2. Let $\mathscr{D}$ be a pure unbounded Hilbert algebra over $\mathscr{D}_{0}$ in a Hilbert space $\mathfrak{S}$. Then $\mathscr{C}(\mathscr{D}), \mathscr{U}\left(L^{\omega}\left(\mathscr{D}_{0}\right)\right)$ and $\mathscr{U}\left(L_{2}^{\omega}\left(\mathscr{D}_{0}\right)\right)$ (resp. $\mathscr{V}(\mathscr{D}), \mathscr{Y}\left(L^{\omega}\left(\mathscr{D}_{0}\right)\right)$ and $\mathscr{V}\left(L_{2}^{\omega}\left(\mathscr{D}_{0}\right)\right)$ ) are pure $E W^{\sharp}$-algebras on $L_{2}^{\omega}\left(\mathscr{D}_{0}\right)$ over $\mathscr{U}_{0}\left(\mathscr{D}_{0}\right)$ (resp. $\mathscr{\mathscr { V }}_{0}\left(\mathscr{D}_{0}\right)$ ). Furthermore, we have

$$
\mathscr{U}\left(L_{2}^{\omega}\left(\mathscr{D}_{0}\right)\right)=\mathscr{\mathscr { C }}\left(L^{\omega}\left(\mathscr{D}_{0}\right)\right), \quad \mathscr{Y}\left(L_{2}^{\omega}\left(\mathscr{D}_{0}\right)\right)=\mathscr{Y}\left(L^{\omega}\left(\mathscr{D}_{0}\right)\right)
$$

and

$$
J \mathscr{U}(\mathscr{D}) J=\mathscr{V}(\mathscr{D}), \quad J \mathscr{V}(\mathscr{D}) J=\mathscr{U}(\mathscr{D}) .
$$

Proof. From ([6] Theorem 3.10) $\mathscr{U}(\mathscr{D}), \mathscr{U}\left(L^{\omega}\left(\mathscr{D}_{0}\right)\right)$ and $\mathscr{U}\left(L_{2}^{\omega}\left(\mathscr{D}_{0}\right)\right)$ are pure $E W^{\sharp}$-algebras on $L_{2}^{\omega}\left(\mathscr{D}_{0}\right)$ over $\mathscr{C}_{0}\left(\mathscr{D}_{0}\right)$. Similarly we can easily prove that $\mathscr{V}(\mathscr{D}), \mathscr{V}\left(L^{\omega}\left(\mathscr{D}_{0}\right)\right)$ and $\mathscr{V}\left(L_{2}^{\omega}\left(\mathscr{D}_{0}\right)\right)$ are pure $E W^{*}$-algebras on $L_{2}^{\omega}\left(\mathscr{D}_{0}\right)$ over $\mathscr{V}_{0}\left(\mathscr{D}_{0}\right)$. We shall show that $\mathscr{U}\left(L^{\omega}\left(\mathscr{D}_{0}\right)\right)=\mathscr{U}\left(L_{2}^{\omega}\left(\mathscr{D}_{0}\right)\right)$. Clearly, $\mathscr{U}\left(L^{\omega}\left(\mathscr{D}_{0}\right)\right) \subset \mathscr{U}\left(L_{2}^{\omega}\left(\mathscr{D}_{0}\right)\right)$. Suppose that $x \in L_{2}^{\omega}\left(\mathscr{D}_{0}\right)$. Let $\overline{\pi_{0}(x)}=U\left|\overline{\pi_{0}(x)}\right|$ be the polar decomposition of $\overline{\pi_{0}(x)}$ and let $\left|\overline{\pi_{0}(x)}\right|=\int_{0}^{\infty} \lambda d E(\lambda)$ be the spectral resolution of $\left|\overline{\pi_{0}(x)}\right|$. Then, $\left|\overline{\pi_{0}(x)}\right|=U^{*} \overline{\pi_{0}(x)}=\overline{\pi_{0}\left(U^{*} x\right)} \in L_{2}^{\omega}\left(\varphi_{0}\right)$. Since $\left|\overline{\pi_{0}(x)}\right|$ is $\varphi_{0}$-restrictedly measurable, $E\left(\lambda_{0}\right)^{\perp} \in L^{2}\left(\varphi_{0}\right)$ for a positive number $\lambda_{0}$. Hence, $\left|\overline{\pi_{0}(x)}\right| E\left(\lambda_{0}\right)^{\perp} \in L_{2}^{\omega}\left(\varphi_{0}\right)\left(L^{2}\left(\varphi_{0}\right) \cap L^{\infty}\left(\varphi_{0}\right)\right) \subset L^{\omega}\left(\varphi_{0}\right)$. Therefore we have

$$
\begin{aligned}
\left|\overline{\pi_{0}(x)}\right| & =\int_{0}^{\lambda_{0}} \lambda d E(\lambda)+\left|\overline{\pi_{0}(x)}\right| E\left(\lambda_{0}\right)^{\perp} \\
& \in \mathscr{U}_{0}\left(\mathscr{D}_{0}\right)+L^{\omega}\left(\varphi_{0}\right) .
\end{aligned}
$$

Hence, $\pi_{2}^{\omega}\left(U^{*} x\right) \in \mathscr{U}\left(L^{\omega}\left(\mathscr{D}_{0}\right)\right)$, and so $\pi_{2}^{\omega}(x) \in \mathscr{L}\left(L^{\omega}\left(\mathscr{D}_{0}\right)\right)$. Consequently $\mathscr{U}\left(L_{2}^{\omega}\left(\mathscr{D}_{0}\right)\right)=\mathscr{U}\left(L^{\omega}\left(\mathscr{D}_{0}\right)\right)$. Similarly we can show that $\mathscr{C}\left(L_{2}^{\omega}\left(\mathscr{D}_{0}\right)\right)=$ 
$\mathscr{V}\left(L^{\omega}\left(\mathscr{D}_{0}\right)\right)$. Since $J \mathscr{U}_{0}\left(\mathscr{D}_{0}\right) J=\mathscr{V}_{0}\left(\mathscr{D}_{0}\right)$ and $\overline{\pi_{0}(x)} J=\overline{\pi_{0}^{\prime}\left(x^{*}\right)}$ for every $x \in \mathscr{D}$, we see that $J \mathscr{U}(\mathscr{D}) J=\mathscr{V}(\mathscr{D})$.

Definition 4.3. $\mathscr{C}(\mathscr{D})$ (resp. $\mathscr{\mathscr { C }}(\mathscr{D})$ ) is called the left (resp. right) $E W^{\sharp}$-algebra of $\mathscr{D}$.

THEOREM 4.4. Let $\mathscr{D}_{0}$ be a Hilbert algebra in a Hilbert space $\mathfrak{S}$ and $\left(\mathscr{D}_{0}\right)_{b} \neq \mathfrak{S}_{\text {. }}$. Then $L_{2}^{\omega}\left(\mathscr{D}_{0}\right)$ is a pure unbounded Hilbert algebra, and $\mathscr{Q}\left(L_{2}^{\omega}\left(\mathscr{D}_{0}\right)\right)$ and $\mathscr{Y}\left(L_{2}^{\omega}\left(\mathscr{D}_{0}\right)\right)$ are pure $E W^{\ddagger}$-algebras on $L_{2}^{\omega}\left(\mathscr{D}_{0}\right)$ over $\mathscr{U}_{0}\left(\mathscr{D}_{0}\right)$ and $\mathscr{V}_{0}\left(\mathscr{D}_{0}\right)$, respectively.

Proof. Theorem 3.4 and Theorem 4.3.

Definition 4.5. Let $\mathfrak{A}$ be an $E W^{\sharp}$-algebra. $\mathfrak{A}$ is called a standard $E W^{\sharp}$-algebra if there exists a pure unbounded Hilbert algebra $\mathscr{D}$ such that $\mathfrak{A}=\mathscr{U}(\mathscr{D})$.

Let $\mathfrak{A}_{0}$ be a semifinite von Neumann algebra on a Hilbert space $\mathfrak{S}$ and let $\varphi_{0}$ be a faithful normal semifinite trace on $\mathfrak{A}_{0}^{+}$. Let $\mathfrak{M}\left(\mathfrak{U}_{0}\right)$ denote the set of all measurable operators with respect to $\mathfrak{A}_{0}$. From ([4] Proposition 4.3) $\mathfrak{M}\left(\mathfrak{A}_{0}\right)$ is an $E W^{*}$-algebra over $\mathfrak{A}_{0}$. Let $\mathfrak{M}_{\varphi_{0}}$ be the maximal ideal associated with $\varphi_{0}$, i.e., $\mathfrak{M}_{\varphi_{0}}=\left\{T \in \mathfrak{A}_{0} ; \varphi_{0}(|T|)<\infty\right\}$. For every $T \in \mathfrak{M}\left(\mathfrak{R}_{0}\right)^{+}$we put

$$
\mu(T)=\sup _{A \in \mathfrak{M}_{\varphi_{0}}^{+}: A \leqq T} \varphi_{0}(A),
$$

and

$$
\begin{aligned}
& L^{p}\left(\varphi_{0}\right)=\left\{T \in \mathfrak{M}\left(\mathfrak{U}_{0}\right) ;\|T\|_{p}:=\mu\left(|T|^{p}\right)^{1 / p}<\infty\right\}, \quad 1 \leqq p<\infty, \\
& L^{\infty}\left(\varphi_{0}\right)=\mathfrak{A}_{0} .
\end{aligned}
$$

Then $L_{2}^{\infty}\left(\varphi_{0}\right):=L^{\infty}\left(\varphi_{0}\right) \cap L^{2}\left(\varphi_{0}\right)$ is a maximal Hilbert algebra in the Hilbert space $L^{2}\left(\varphi_{0}\right)$ under the inner product $(S \mid T)=\mu\left(T^{*} \cdot S\right)$ and $L_{2}^{\omega}\left(\varphi_{0}\right):=\bigcap_{2 \leqq p<\infty} L^{p}\left(\varphi_{0}\right)$ is a maximal unbounded Hilbert algebra over $L_{2}^{\infty}\left(\varphi_{0}\right)$. Let $\mathscr{D}\left(\varphi_{0}\right)$ be an unbounded Hilbert algebra in $L^{2}\left(\varphi_{0}\right)$ over $L_{2}^{\infty}\left(\varphi_{0}\right)$. Then $\mathscr{D}\left(\varphi_{0}\right)$ is regarded as a $*$-algebra on $\mathscr{F}$ under the strong sum, strong product, adjoint and strong scalar multiplication. We denote by $\mathfrak{Z}\left(\mathscr{D}\left(\varphi_{0}\right)\right)$ the set of closed operators on $\mathscr{F}$ which is the *-algebra generated by $\mathscr{D}\left(\varphi_{0}\right)$ and $\mathfrak{A}_{0}$. Then $\mathfrak{A}\left(\mathscr{D}\left(\varphi_{0}\right)\right)$ is an $E W^{*}$-algebra over $\mathfrak{A}_{0}$ and it is isomorphic to the left $E W^{\sharp}$-algebra $\mathscr{U}\left(\mathscr{D}\left(\varphi_{0}\right)\right)$.

THEOREm 4.5. Let $\mathfrak{A}_{0}$ be a semifinite von Neumann algebra on a Hilbert space $\mathfrak{S}$ and let $\varphi_{0}$ be a faithful normal semifinite trace on 
$\mathfrak{A}_{0}^{+}$. If $L^{2}\left(\varphi_{0}\right)$ is not a Hilbert algebra, i.e., $L^{2}\left(\varphi_{0}\right) \neq L_{2}^{\infty}\left(\varphi_{0}\right)$, then there exists a pure $E W^{*}$-algebra $\mathfrak{A}$ over $\mathfrak{A}_{0}$ such that is isomorphic to a standard EW $E W^{\sharp}$ algebra. In particular, if $\cap_{T \in L_{2}^{\omega}\left(\varphi_{0}\right)} \mathscr{D}(T)$ is dense in $\mathfrak{\mathcal { B }}$, then we may regard $\mathfrak{A}$ as a pure EW $W^{\sharp}$-algebra over $\mathfrak{A}_{0}$.

CoROLlary 4.6. Let $\mathfrak{A}_{0}$ be a semifinite von Neumann algebra on a Hilbert space $\mathfrak{S}$ and let $\varphi_{0}$ be a faithful normal semifinite trace on $\mathfrak{A}_{0}^{+}$. If $\mathfrak{A}$ is a pure EW*-algebra over $\mathfrak{A}_{0}$ such that $\mathfrak{A} \subset$ $\mathfrak{A}\left(L_{2}^{\omega}\left(\varphi_{0}\right)\right)$, then $\mathfrak{A}$ is isomorphic to a standard $E W^{\sharp}$-algebra.

Proof. We can easily prove that $\mathfrak{A} \cap L_{2}^{\omega}\left(\varphi_{0}\right)$ is a pure unbounded Hilbert algebra over $L_{2}^{\infty}\left(\varphi_{0}\right)$ and $\mathfrak{A}$ is isomorphic to $\mathscr{C}\left(\mathfrak{U} \cap L_{2}^{\omega}\left(\varphi_{0}\right)\right)$.

\section{REFERENCES}

1. W. Ambrose, The $L^{2}$-system of a unimodular group, Trans. Amer. Math. Soc., 65 (1949), 27-48.

2. R. Arens, The space $L^{\omega}$ and convex topological rings, Bull. Amer. Math. Soc., 52 (1946), 931-935.

3. J. Dixmier, Les algêbres d'opérateurs dans l'espace Hilbertian, Gaushier-Villars, Paris, 2é edition (1969).

4. P. G. Dixon, Unbounded operator algebras, Proc. London Math. Soc., (3) 21 (1970), 693-715.

5. A. Inoue, On a class of unbounded operator algebras, Pacific J. Math., 65 (1976), 77-95.

6. - On a class of unbounded operator algebras II, Pacific J. Math., (to appear).

7. — Unbounded Hilbert algebras as locally convex *algebras, Math. Rep. College of General Edu. Kyushu-Univ., X(2) (1976), 279-293.

8. T. Ogasawara and K. Yoshinaga, A noncommutative theory of integration for operators, J. Sci. Hiroshima Univ., 18 (3) (1955), 311-347.

9. R. Pallu de La Barrière, Algèbres unitaires et espaces d'Ambrose, Ann. ÉC. Norm. Sup., 70 (1953), 381-401.

10. I. E. Segal, A noncommutative extension of abstract integration, Ann. Math., 57 (1953), 401-457.

Received June 16, 1976 and in revised form September 28, 1976.

FUkUOKa UNIVERSITy 



\section{PACIFIC JOURNAL OF MATHEMATICS}

EDITORS

RICHARD ARENS (Managing Editor)

University of California

Los Angeles, California 90024

R. A. Beaumont

University of Washington

Seattle, Washington 98105
J. DUGUNDJI

Department of Mathematics

University of Southern Californı

Los Angeles, California 90007

D. Gilbarg and J. Milgram

Stanford University

Stanford, California 94305

\section{ASSOCIATE EDITORS}

E. F. BECKENBACH

B. H. NeumanN

F. WOLF

K. YoSHIDA

\section{SUPPORTING INSTITUTIONS}

UNIVERSITY OF BRITISH COLUMBIA

CALIFORNIA INSTITUTE OF TECHNOLOGY

UNIVERSITY OF CALIFORNIA

MONTANA STATE UNIVERSITY

UNIVERSITY OF NEVADA

NEW MEXICO STATE UNIVERSITY

OREGON STATE UNIVERSITY

UNIVERSITY OF OREGON

OSAKA UNIVERSITY
UNIVERSITY OF SOUTHERN CALIFORNIA

STANFORD UNIVERSITY

UNIVERSITY OF TOKYO

UNIVERSITY OF UTAH

WASHINGTON STATE UNIVERSITY

UNIVERSITY OF WASHINGTON

AMERICAN MATHEMATICAL SOCIETY

NAVAL WEAPONS CENTER 


\section{Pacific Journal of Mathematics}

Vol. 69 , No. 1

May, 1977

V. V. Anh and P. D. Tuan, On starlikeness and convexity of certain analytic

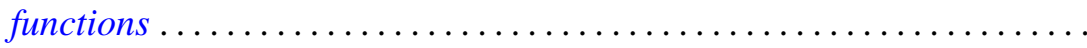

Willard Ellis Baxter and L. A. Casciotti, Rings with involution and the prime

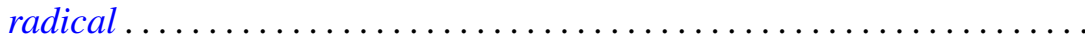

Manuel Phillip Berriozabal, Hon-Fei Lai and Dix Hayes Pettey,

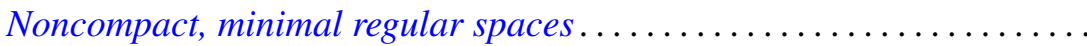

Sun Man Chang, Measures with continuous image law ................

John Benjamin Friedlander, Certain hypotheses concerning

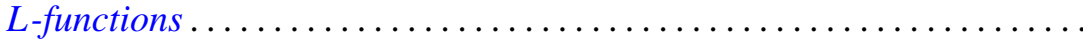

Moshe Goldberg and Ernst Gabor Straus, On characterizations and

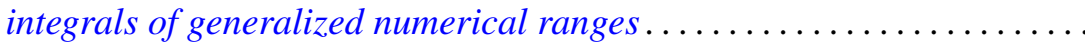

Pierre A. Grillet, On subdirectly irreducible commutative semigroups ...... 55

Robert E. Hartwig and Jiang Luh, On finite regular rings ..............

Roger Hugh Hunter, Fred Richman and Elbert A. Walker, Finite direct sums of cyclic valuated p-groups ........................... 97

Atsushi Inoue, On a class of unbounded operator algebras. III ......... 105

Wells Johnson and Kevin J. Mitchell, Symmetries for sums of the Legendre

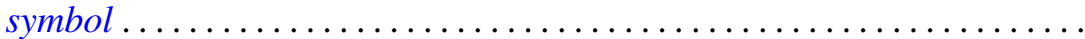

Jimmie Don Lawson, John Robie Liukkonen and Michael William Mislove,

Measure algebras of semilattices with finite breadth

Glenn Richard Luecke, A note on spectral continuity and on spectral properties of essentially $G_{1}$ operators ...............

Takahiko Nakazi, Invariant subspaces of weak-* Dirichlet algebras . .

James William Pendergrass, Calculations of the Schur group ...

Carl Pomerance, On composite $n$ for which $\varphi(n) \mid n-1$. II. . .

Marc Aristide Rieffel and Alfons Van Daele, A bounded operator approach to Tomita-Takesaki theory........................

Daniel Byron Shapiro, Spaces of similarities. IV. $(s, t)$-families ...

Leon M. Simon, Equations of mean curvature type in 2 independent variables.

Joseph Nicholas Simone, Metric components of continuous images of ordered compacta ............................

William Charles Waterhouse, Pairs of symmetric bilinear forms in

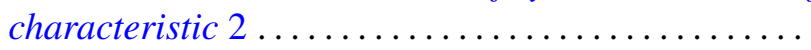

